\title{
Upper Extremity Catheter Angiography: Indications, Techniques, Anatomy, and Classic Cases
}

\author{
David S. Shin ${ }^{1} \quad$ David B. Magill ${ }^{1}$ \\ Eric J. Monroe ${ }^{1}$ \\ ${ }^{1}$ Section of Interventional Radiology, Department of Radiology, \\ University of Washington, Seattle, Washington, United States
}

\begin{abstract}
Address for correspondence Eric J. Monroe, MD, Department of Radiology, Seattle Children's Hospital, 4800 Sand Point Way NE, M/S R-5417, Seattle, WA 98105, United States

(e-mail: eric.monroe@seattlechildrens.org).
\end{abstract}

\begin{abstract}
Keywords

- upper extremity

- artery

- angiography

Catheter arteriography of the upper extremity has become an infrequent procedure in modern practice given the advancements in computed tomography/magnetic resonance angiography (CT/MRA). However, catheter angiography continues to have major diagnostic and interventional roles in certain pathophysiologic conditions. This article discusses the indications and techniques of upper extremity catheter angiography, and presents classic cases to demonstrate key anatomic, diagnostic, and interventional considerations.
\end{abstract}

\section{Introduction}

Catheter angiography remained the gold standard for evaluation of upper extremity arterial pathology until succeeded by computed tomography angiography ${ }^{1}$ (CTA) and magnetic resonance angiography (MRA). Although its scope has narrowed, it continues to play an important role in the evaluation and management of trauma, limb ischemia, hemodialysis access, vasculitis, and vascular anomalies.

Herein we review the modern indications of upper extremity catheter angiography, patient preparation and angiographic techniques, normal and variant anatomy, and classic angiographic diagnoses.

\section{Indications}

\section{Trauma}

Evaluation of upper extremity injury begins with rapid physical examination to assess for signs of major arterial injury, such as expanding hematoma, pulsatile bleeding, palpable thrill or audible bruit, and profound distal ischemia. ${ }^{2}$ Evaluation for injuries not definitive on physical examination may involve Doppler ultrasound and/or CTA. Catheter angiography is a useful problem-solving tool when CTA is inconclusive. Furthermore, suspicious findings on the noninvasive imaging may prompt angiography for operative planning or definitive endovascular intervention.

received

January 18, 2018

accepted after revision

April 4, 2018

published online

July 3, 2018
Common traumatic arterial injuries include pseudoaneurysm, dissection, thrombosis, transection, and arteriovenous fistula ( $\mathbf{- F i g s . 1 - 3}$ ), and may occasionally be amenable to endovascular treatment. In a small retrospective study, Carrafiello et al reported high technical success and short-term clinical success rates for treating pseudoaneurysms and transections with stent grafts and treating dissections and mural hematomas with bare stents or angioplasty. ${ }^{3}$ Long-term patency rates in this setting are not well established. Endovascular intervention for upper extremity trauma is rare in clinical practice, and surgical management remains standard of care especially in penetrating trauma.

\section{Acute Limb Ischemia}

Acute limb ischemia presents with varying degrees of severity (-Table 1), ranging from a viable limb with intact sensorimotor functions to an irreversible ischemic damage with profound sensorimotor deficits. ${ }^{4}$ Those presenting with severe acute ischemia (i.e., Rutherford category IIb or III) should undergo emergent surgical revascularization or amputation.

Catheter-directed thrombolysis for acute thromboembolism in the upper extremity is an attractive alternative to surgical embolectomy in patients without immediate threat of limb loss. ${ }^{5,6}$ These cases typically include patients with Rutherford category I and IIa acute ischemia., ${ }^{4,7}$ Common clinical situations include distal embolism from the 


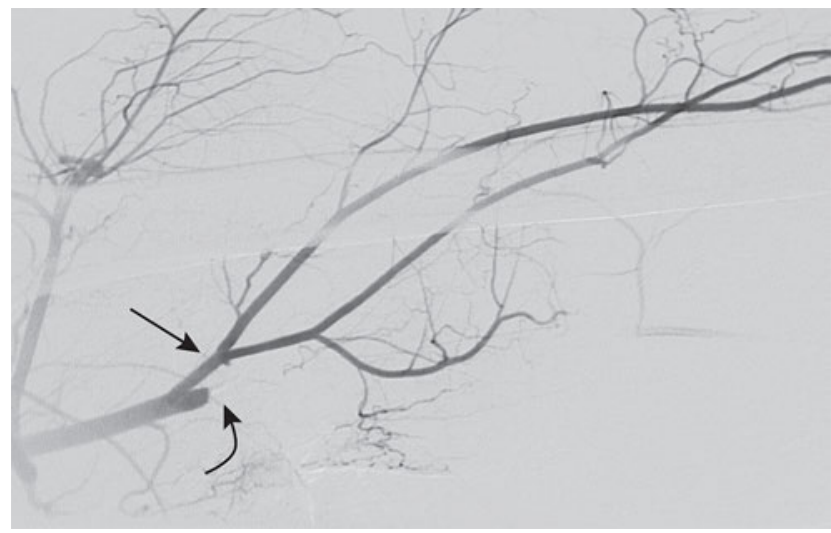

Fig. 1 Brachial artery traumatic occlusion. An avulsion injury was sustained during waterskiing, and the patient presented with diminished peripheral left upper extremity pulses. Emergent angiographic evaluation revealed occlusion of the proximal brachial artery (curved arrow). Robust profunda brachii branches are visualized (straight arrow).
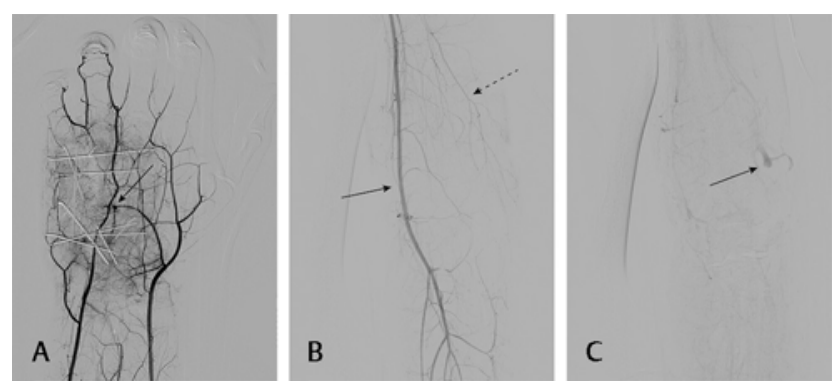

Fig. 2 Penetrating trauma arterial injuries. (A) Gunshot wound to the hand resulting in pseudoaneurysm and transection of the deep palmar arch (arrow). Multiple surgical fixation wires are noted. (B) Brachial arteriogram after penetrating trauma to the upper extremity demonstrates a patent brachial artery (arrow) and profunda brachii muscular branch (dashed arrow). (C) While a discrete arteriovenous connection was not identified, a dilated early draining cephalic vein (arrow) in the territory of the muscular branches suggested shunting through small traumatic fistulas.
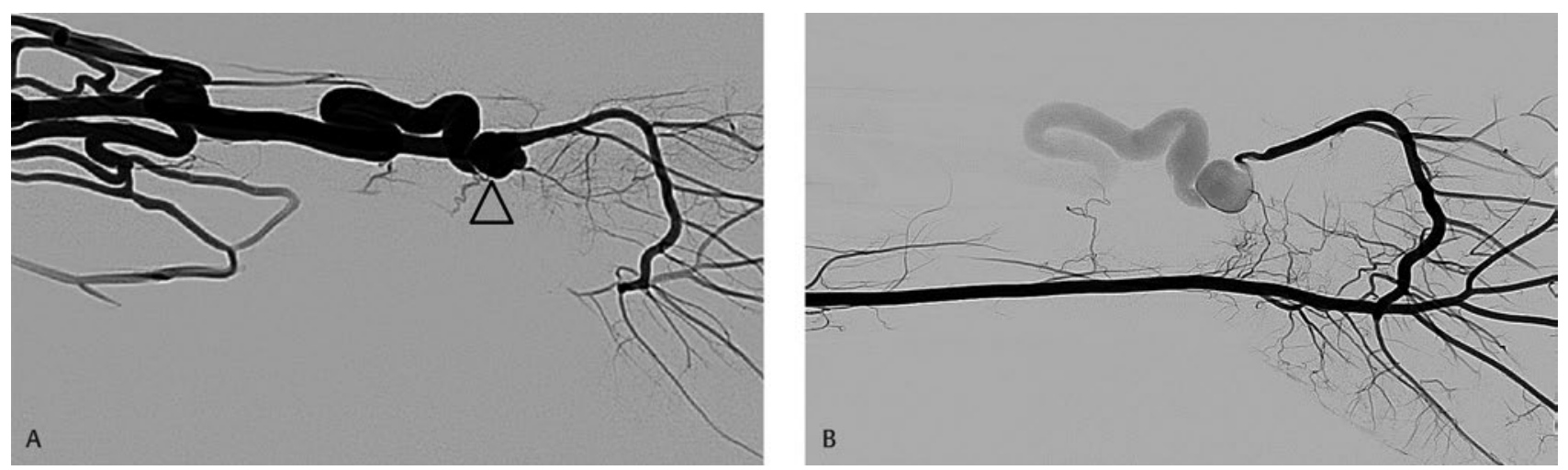

Fig. 3 latrogenic trauma-arteriovenous fistula postradial artery access. (A) Radial artery angiogram demonstrates a fistulous connection between the radial artery and cephalic vein (arrowhead). (B) Ulnar artery angiogram injection confirms the previous finding with steal phenomenon through the palmar arch.

Table 1 Rutherford classification for acute limb ischemia ${ }^{4}$

\begin{tabular}{|c|c|c|c|c|c|}
\hline \multirow[t]{2}{*}{ Category } & \multirow[t]{2}{*}{ Description/Prognosis } & \multicolumn{2}{|c|}{ Findings } & \multicolumn{2}{|c|}{ Doppler signal } \\
\hline & & Sensory deficit & Motor deficit & Arterial & Venous \\
\hline I. Viable & Not immediately threatened & None & None & Audible & Audible \\
\hline \multicolumn{6}{|l|}{ II. Threatened } \\
\hline a. Marginally & Salvageable if promptly treated & None or minimal & None & Inaudible & Audible \\
\hline b. Immediately & $\begin{array}{l}\text { Salvageable with immediate } \\
\text { revascularization }\end{array}$ & $\begin{array}{l}\text { Mild or } \\
\text { moderate }\end{array}$ & $\begin{array}{l}\text { Mild or } \\
\text { moderate }\end{array}$ & Inaudible & Audible \\
\hline III. Irreversible & $\begin{array}{l}\text { Major tissue loss or permanent } \\
\text { nerve damage inevitable }\end{array}$ & $\begin{array}{l}\text { Profound } \\
\text { (anesthetic) }\end{array}$ & $\begin{array}{l}\text { Profound } \\
\text { (paralysis) }\end{array}$ & Inaudible & Inaudible \\
\hline
\end{tabular}

subclavian artery injury in the setting of arterial thoracic outlet syndrome ( - Fig. 4 ) and cardiogenic thromboembolism (-Fig. 5). Standard absolute and relative contraindications to pharmacologic thrombolysis apply (- Table 2$).{ }^{8}$ An infusion catheter is positioned across or just proximal to the site of occlusion, with an initial bolus of 3 to $5 \mathrm{mg} \mathrm{t}-\mathrm{PA}$, followed by infusion at 0.5 to $1 \mathrm{mg} / \mathrm{h}$. Systemic heparin can be concomitantly administered through the arterial sheath for a goal activated partial thromboplastin time (aPTT) of 60 to 80 seconds. Compared with lower extremity thrombolysis, risks are considerable and include distal embolization of loss of digits and brachial sheath hematoma with neurologic injury; thrombolysis is best considered in an interdisciplinary fashion and weighed against the feasibility and risks of surgical embolectomy or conservative management with anticoagulation alone. Patients undergoing thrombolysis are monitored in the intensive care unit for clinical improvement and hemorrhagic complications. Repeat angiography after 12 to 24 hours of thrombolysis assesses for restoration of flow and presence of an underlying lesion. 

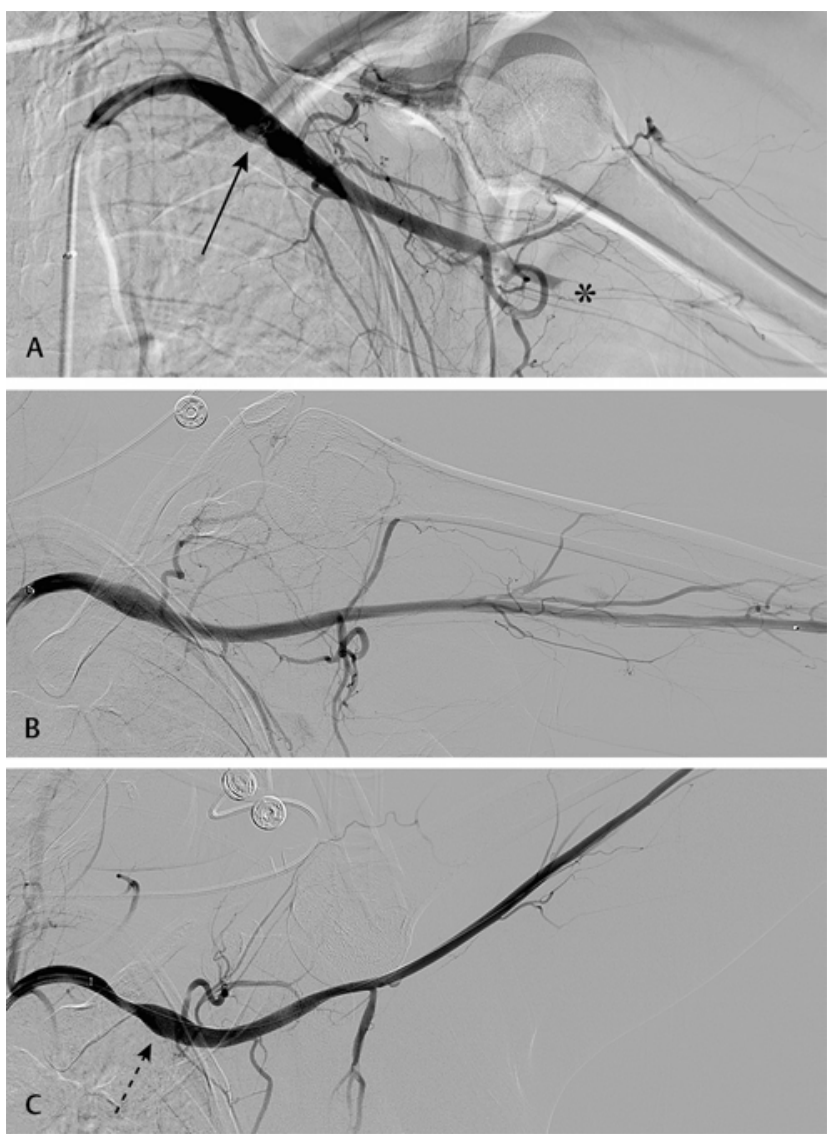

Fig. 4 Arterial thoracic outlet syndrome. (A) Left subclavian arteriogram demonstrates aneurysmal dilation and irregular filling defects at the distal subclavian artery (arrow). The proximal brachial artery (asterisk) is occluded from a large embolus. (B) Overnight catheter-directed thrombolysis using tPA infusion restored brachial arterial flow. (C) Repeat subclavian arteriogram with arm abduction accentuates the arterial compression. Poststenotic dilation (dashed arrow) is typical of arterial thoracic outlet syndrome.
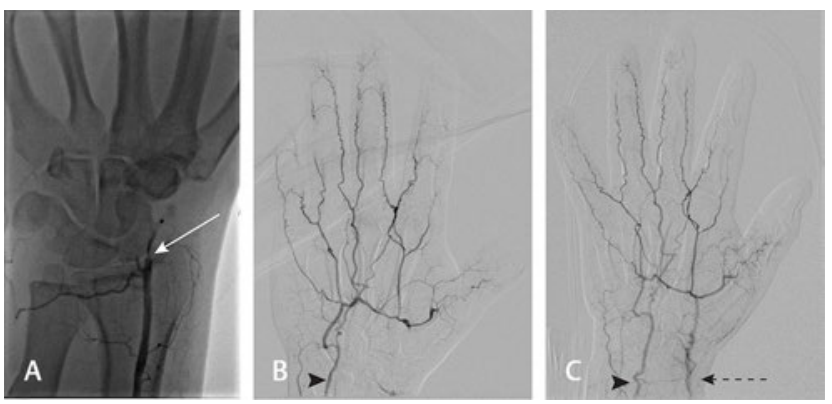

Fig. 5 Cardiac embolism in atrial fibrillation. (A) Selective radial arteriogram demonstrates occlusion at the radiocarpal joint (arrow) due to embolus in a patient with atrial fibrillation. (B) Hand arteriogram demonstrates preserved ulnar arterial flow (arrowhead) with complete superficial palmar arch that supplies all the digits. (C) Repeat hand arteriogram after catheter-directed thrombolysis demonstrates restored flow in the radial artery (dashed arrow).

\section{Bypass Graft Planning}

The radial artery is a frequently used arterial conduit for coronary artery bypass surgery ${ }^{9}$ and extracranial-intracranial bypass surgery. Upper extremity catheter angiography is often performed in conjunction with coronary or carotid angiography

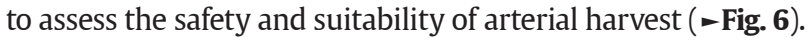

Table 2 Contraindications to pharmacologic thrombolysis ${ }^{8}$

\begin{tabular}{|l|}
\hline Absolute \\
\hline $\begin{array}{l}\text { - Established cerebrovascular event (including TIAs) within } \\
\text { past } 2 \text { mo }\end{array}$ \\
\hline - Active bleeding diathesis \\
\hline - Gastrointestinal bleeding within past $10 \mathrm{~d}$ \\
\hline - Neurosurgery (intracranial, spinal) within past 3 mo \\
\hline - Intracranial trauma within past 3 mo \\
\hline Relative \\
\hline - Cardiopulmonary resuscitation within past $10 \mathrm{~d}$ \\
\hline - Major nonvascular surgery or trauma within past $10 \mathrm{~d}$ \\
\hline - Uncontrolled hypertension: systolic > $180 \mathrm{~mm} \mathrm{Hg} \mathrm{or}$ \\
diastolic > 110 mm Hg \\
\hline - Puncture of noncompressible vessel \\
\hline - Intracranial tumor \\
\hline - Recent eye surgery
\end{tabular}

Abbreviation: TIA, transient ischemic attack.

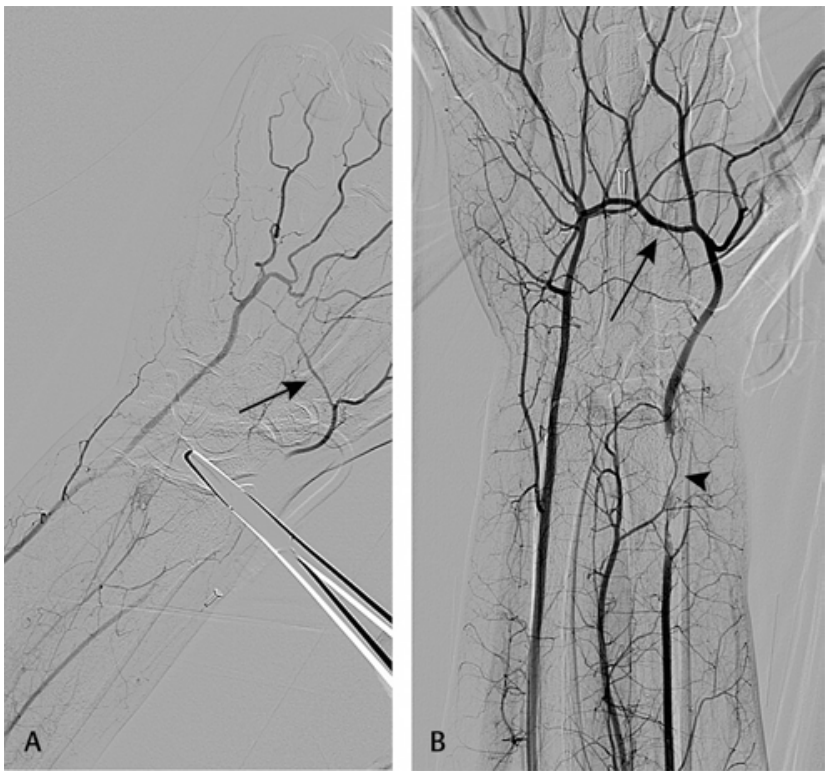

Fig. 6 Preoperative catheter angiography for radial artery harvesting. (A) Angiographic Allen's test. Clamp compression of the radial artery confirms complete deep palmar arch (arrow) filling from the ulnar artery. This radial artery can be safely ligated and harvested. (B) Angiography in a second patient demonstrates segmental occlusion of the distal radial artery (arrowhead), asymptomatic and attributed to prior arterial line access. Note the complete superficial palmar arch from the ulnar artery (arrow).

\section{Dialysis Access Steal Syndrome}

Symptomatic hand ischemia in patients with dialysis access ("steal syndrome") is an infrequent but serious condition. Signs and symptoms include paresthesia, weakened pulses, pain, muscle atrophy, paralysis, decreased skin temperature, cyanosis, and gangrene. Multiple coexisting etiologies, including retrograde flow distal to the anastomosis, arterial inflow stenosis, and distal arteriopathy, contribute. ${ }^{10}$ When suspected, the workup begins with Doppler ultrasound. For severe symptoms or digital 


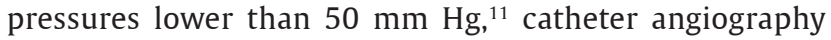
can confirm reversal of flow ( - Fig. 7 ), distal arteriopathy, and/or presence of inflow stenosis.

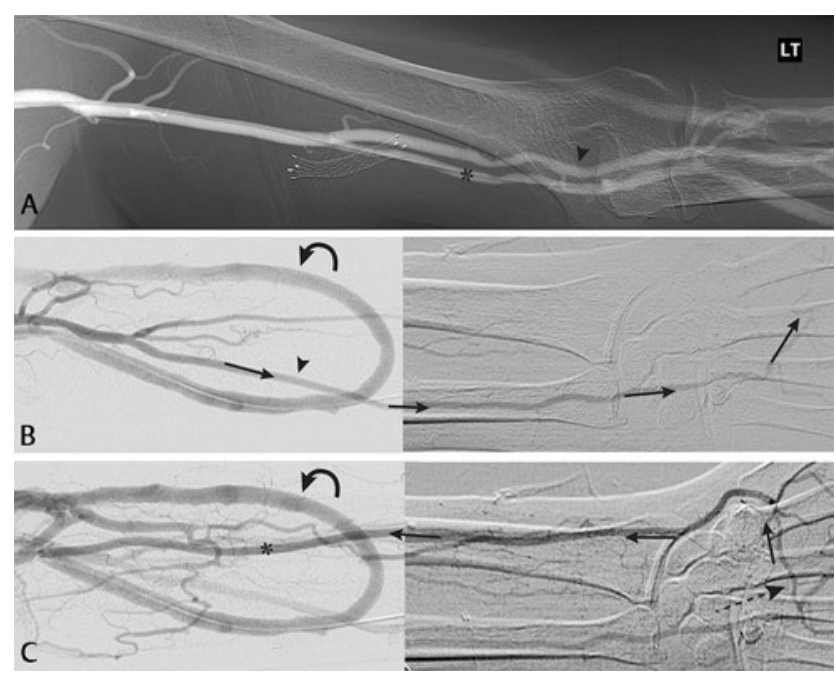

Fig. 7 Dialysis access steal phenomenon. (A) Brachial arteriogram demonstrates early branching of the radial (asterisk) and ulnar arteries (arrowhead). (B) Dialysis arteriovenous loop graft (curved arrow) is anastomosed with the radial artery. The graft is widely patent. There is antegrade flow in the ulnar artery (arrows denote direction of flow). No antegrade flow is seen along the radial artery. (C) The ulnar artery retrogradely supplies the radial artery via the deep palmar arch (dashed arrow), confirming true steal physiology.

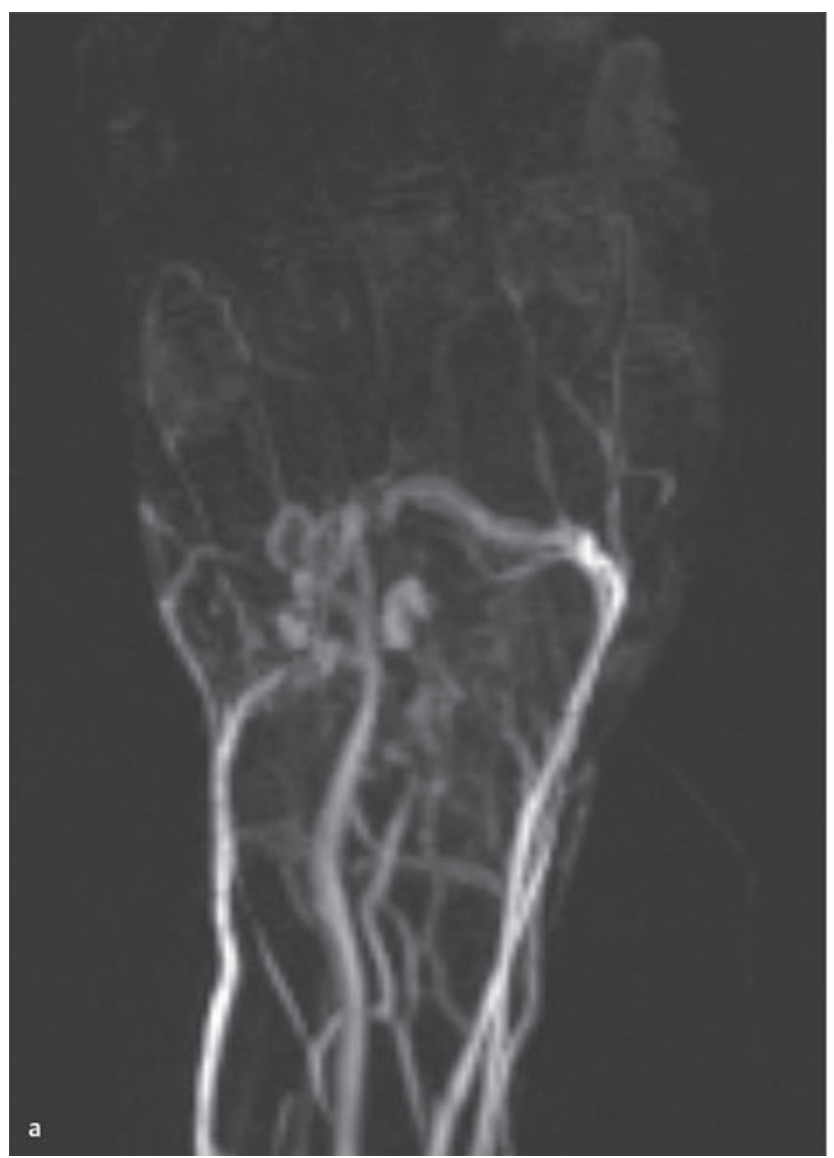

\section{Vasculitis}

Laboratory analysis typically precedes imaging for suspected vasculitis. While the presence and concentrations of certain serologic markers may have adequate specificity for diagnosis, angiography is often a helpful adjunctive method. While high-quality CTA/MRA are often adequate to evaluate medium- and large-vessel vasculitides, small-vessel vasculitides (e.g., microscopic polyangiitis, granulomatosis with polyangiitis, Churg-Strauss syndrome) are better assessed with the superior spatial resolution of catheter angiography. ${ }^{12}$

\section{Vascular Anomalies}

Catheter angiography is considered when CTA or MRA fails to adequately demonstrate arterial feeder(s) of arteriovenous fistulas and malformations. It can clarify the anatomy of the lesion prior to endovascular embolization or surgery (-Fig. 8). Angiography also plays an important role in preoperative embolization of vascular tumors involving the upper extremity, such as sarcomas and hypervascular metastases, with intention to decrease operative blood loss.

\section{Techniques}

\section{Preparation and Patient Positioning}

Equipment must be able to accommodate imaging of the entire upper extremity (aortic arch to fingertips). For suspected

Fig. 8 Arteriovenous malformation. (A) Time-resolved magnetic resonance angiography image of the right hand demonstrates an arteriovenous malformation at the ulnar aspect of the wrist. (B) Angiogram confirms the previous finding with excellent spatial detail confirming complex supply from radial, ulnar, interosseous, and arch arteries. 
vasculitis, catheter angiography of the clinically unaffected upper extremity, lower extremities, and mesenteric arteries should be considered to confirm and evaluate systemic involvement. For compression syndromes (discussed below), patient positioning should allow for provocative maneuvers without disrupting the sterile barrier.

The arterial vasculature of the hand is highly sensitive to various stimuli commonly found in the angiography suite, including cold temperature, pressure/restraints, pain, and anxiety..$^{13}$ These should be eliminated or mitigated with heat packs, warm blankets, sedation, and careful positioning to avoid false-positive examinations.

Patient cooperation and comfort minimize motion artifacts. Position the hand flat on a padded arm board in anatomic position (i.e., palm up) with the digits slightly spread out to avoid overlapping vessels. Tight restraints or heavy hot packs should be avoided to minimize direct pressure on the arteries.

\section{Equipment and Access}

The arch aortogram is performed via a pigtail flush catheter in a 30-degree left anterior oblique projection (i.e., "candy cane" view), at a $15-$ to $30-\mathrm{mL} / \mathrm{s}$ injection rate for a total of 30 to $50 \mathrm{~mL}$ of contrast agent, and with at least three to four frames per second imaging acquisition. A 100 - to $125-\mathrm{cm}, 4$ or $5 \mathrm{~F}$ angled catheter (e.g., Vertebral, Berenstein, or Headhunter) is used to select the target subclavian artery ( - Fig. 9). In cases of tortuous and ectatic arches, reverse curve catheters (e.g., Simmons) may be useful. The subclavian arteriography is performed in the anteroposterior (AP) projection, typically at a 5- to $7-\mathrm{mL} / \mathrm{s}$ injection rate for a total of 10 to $15 \mathrm{~mL}$ of contrast agent, and with two frames per seconds imaging acquisition. Arteriography of the remaining upper extremity is performed in multiple stations. Further selection of the brachial artery may improve image quality and reduce contrast bolus volumes. Decreased frame rate (e.g., one to two frames per seconds) reduces radiation in the setting of diseased distal arteries with slow flow.

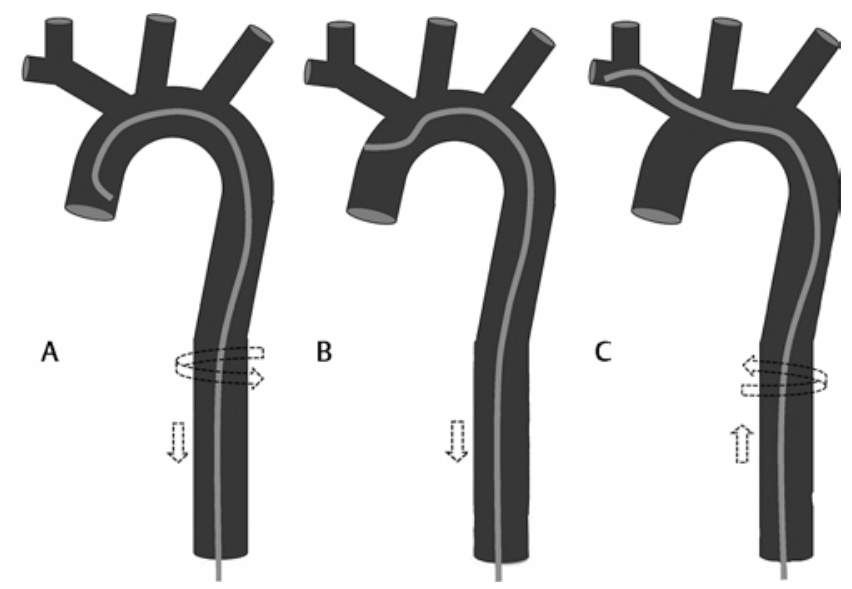

Fig. 9 Right subclavian artery catheterization technique. A vertebral catheter is advanced into the ascending aorta with the curve of the catheter matching the aorta. The catheter is turned counterclockwise for cephalad orientation of the catheter tip and slowly withdrawn until the brachiocephalic artery is selected. The catheter is then turned clockwise while being slowly advanced during gentle manual contrast injection until the right subclavian artery is selected.

\section{Vasodilators}

Available agents for prevention and reversal of vasospasm include nifedipine (10 $\mathrm{mg}$ SL or PO), verapamil (2.5-5 mg intra-arterial), nitroglycerine (100-200 $\mu \mathrm{g}$ intraarterial or $1-2$ in [7.5-15 $\mathrm{mg}$ ] transdermal paste), papaverine (60 mg intra-arterial), and tolazoline ( $25 \mathrm{mg}$ intra-arterial). Agents may be combined for maximum result, while closely monitoring the systemic blood pressure.

\section{Diagnosis}

\section{Anatomy and Variants}

The aortic arch conventionally gives rise to the brachiocephalic artery, left common carotid artery, and left subclavian artery (-Fig. 10). Arch variations occur in approximately $17 \%$, most frequently a common origin of the brachiocephalic and left common carotid arteries (15\%). ${ }^{14}$

The brachiocephalic artery gives rise to the right subclavian artery and the right common carotid artery. The subclavian artery extends to the lateral border of the first rib, where it continues as the axillary artery (-Fig. 11). Branch patterns of the subclavian artery are variable, but they include the vertebral artery, internal thoracic artery, thyrocervical trunk, and costocervical trunk. The axillary artery extends from the lateral border of the first rib to the lateral border of the teres minor muscle. The major branches of the axillary artery include thoracoacromial, lateral thoracic, subscapular, and circumflex humeral arteries. The axillary artery continues as the brachial artery in the upper extremity (-Fig. 11). The profunda brachii is the largest branch of the brachial artery, supplying muscular branches to the posterior upper arm. Collateral pathways at the elbow are named for their anastomotic connections (e.g., radial and ulnar recurrent arteries). The brachial artery terminally divides into the ulnar and radial arteries within the inferior aspect of the cubital fossa (-Fig. 12). The ulnar artery gives rise proximally to the interosseous artery.
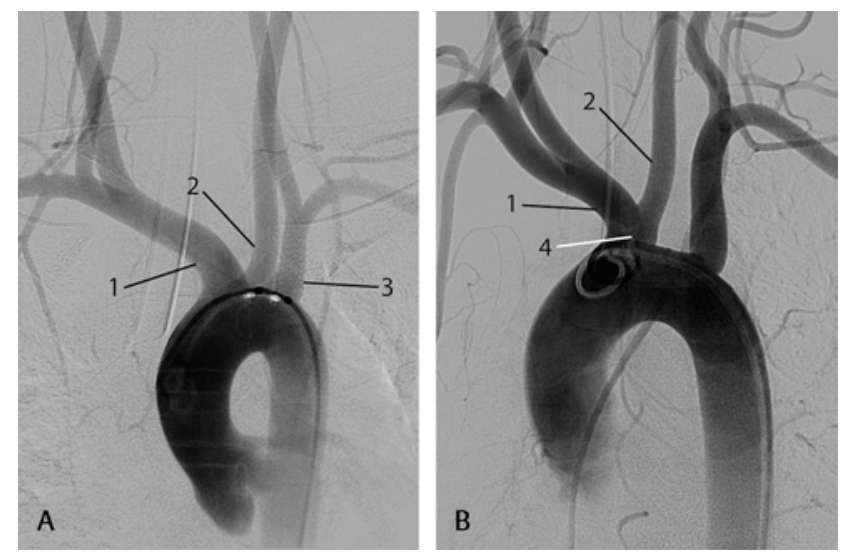

Fig. 10 Aortic arch anatomy. (A) Conventional aortic arch. There are three distinct origins of the great vessels, including the brachiocephalic (innominate) artery (1), left common carotid artery (2), and left subclavian artery (3). (B) Bovine aortic arch. This is the most common variant of the arch with a common truncus (4) for the brachiocephalic (1) and left common carotid (2) arteries. The left subclavian artery origin remains separate. 

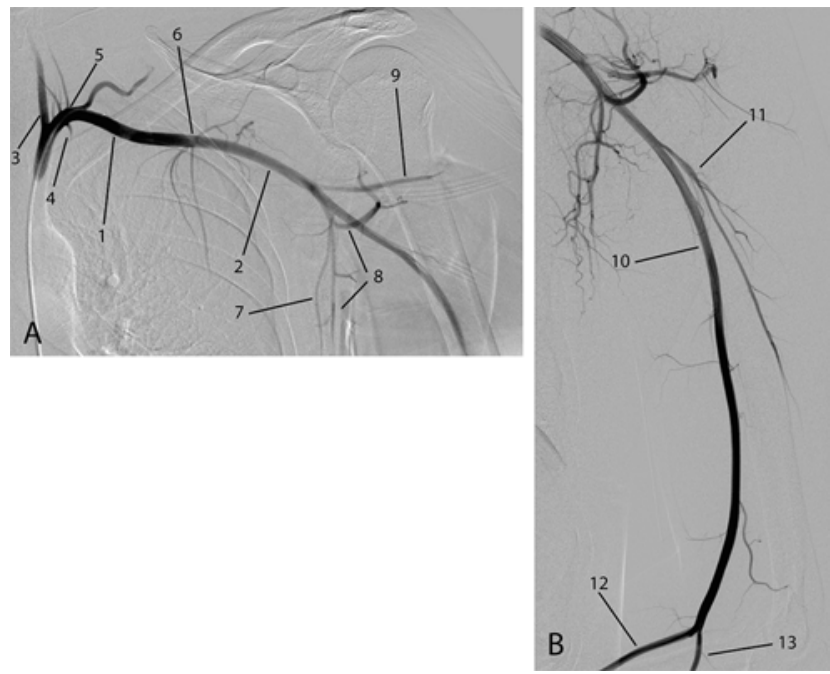

Fig. 11 Subclavian, axillary, and brachial artery conventional anatomy. (A) The subclavian artery (1) gives off the vertebral artery (3), internal thoracic artery (4), and thyrocervical trunk (5). The axillary artery (2) gives off the thoraco-acromial (6), lateral thoracic (7), subscapular (8), circumflex scapular and thoracodorsal branches), and circumflex humeral arteries (9). (B) The brachial artery (10) gives off profunda brachii (11) before bifurcating near the elbow into the radial (12) and ulnar (13) arteries.

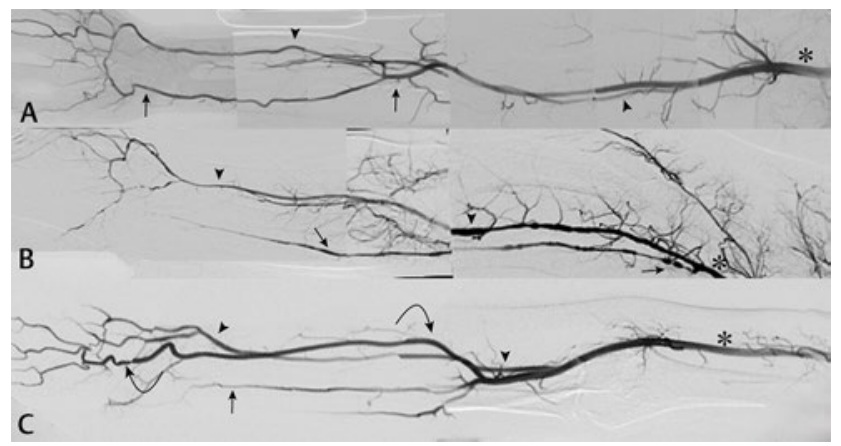

Fig. 13 Brachial artery variant anatomy. (A) High origin of the radial artery (arrowhead) from the brachial artery (asterisk) in the upper arm. (B) High origin of the ulnar artery (arrow) from the brachial artery (asterisk) in the upper arm. Note the multifocal narrowing and irregularity of the ulnar and radial arteries in this patient with traumatic humeral fracture resulting in diffuse stretch injury and vasospasm. (C) Persistent median artery (curved arrow) supplying significant flow to the hand. Normal radial artery (arrowhead) is seen, whereas the ulnar artery (arrow) is diminutive.

Common anatomic variants of the arm and forearm arteries include early origin of the radial or ulnar arteries ( - Fig. 13A, B). This so-called high origin of the radial artery is relatively common, occurring in up to $14 \%$ of patients, while a high origin of the ulnar artery is much less frequent, occurring in 0.17 to $2 \% .{ }^{15,16}$ Continuation of the interosseous artery as a distinct third vessel entering the hand, termed a persistent median artery ( - Fig. 13C) is rare and may cause compressive neuropathy involving the median nerve. ${ }^{17}$

Arterial supply to the hand consists of multiple complex anastomotic networks in the palmar and dorsal aspects of
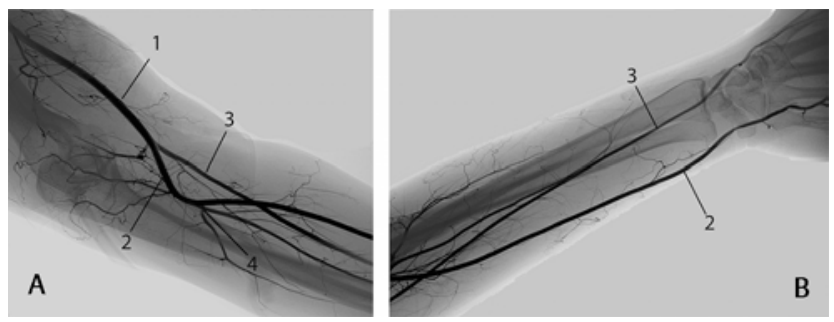

Fig. 12 Forearm artery conventional anatomy. (A) The distal brachial artery (1) bifurcates at the inferior aspect of the cubital fossa into the ulnar (2) and radial (3) arteries. The ulnar artery characteristically gives rise to the interosseous artery (4). (B) The ulnar and radial arteries course along the forearm and enter the hand.
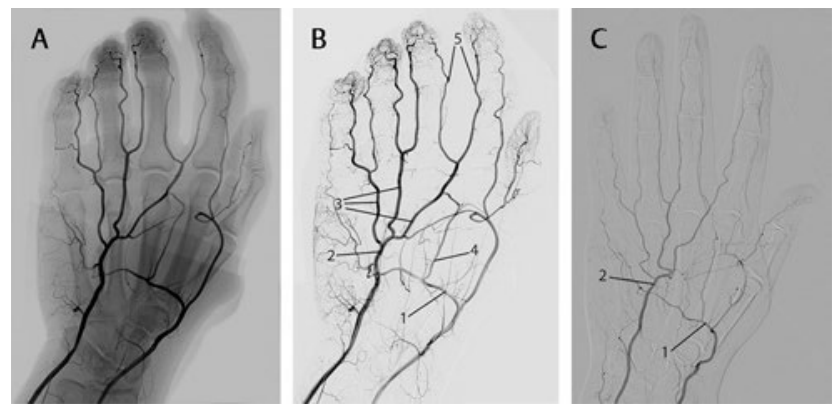

Fig. 14 Hand artery conventional anatomy. Native (A) and digital subtraction (B) angiograms of the hand. The radial artery supplies the deep palmar arch (1), and the ulnar artery supplies the superficial palmar arch (2). At the level of the mid metacarpals, the superficial palmar arch gives rise to common palmar digital arteries (3), which are joined by palmar metacarpal arteries (4) originating from the deep palmar arch. At the web space, the common palmar digital artery splits into two proper palmar digital arteries (5) to supply the adjacent fingers. (C) Hand angiogram in another patient. Note the diminutive and nearly incomplete superficial palmar arch (2) compared with the distinct deep palmar arch (1).

the hand, formed by branches of the ulnar and radial arteries (-Fig. 14). The palmar component is based on two palmar arches: superficial (distal and supplied by the ulnar artery) and deep (proximal and supplied by the radial artery). These arches are frequently incomplete, particularly the superficial palmar arch where a true arcade is only

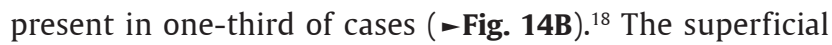
palmar arch gives rise to the common palmar digital arteries, which join the palmar metacarpal arteries originating from the deep palmar arch. At the web space, the common palmar digital artery splits into two proper palmar digital arteries to supply the adjacent fingers.

\section{Classic Angiographic Cases \\ Takayasu's Arteritis}

Takayasu's arteritis is a large-vessel vasculitis commonly affecting the aorta and its branch vessels and frequently occurs in young women of Southeast Asian and Indian descent. ${ }^{19}$ Smoothly tapering stenoses or complete occlusions involving major aortic branch vessels are typical (-Fig. 15), frequently with extensive collateralization if long standing. Aneurysms are infrequent. 

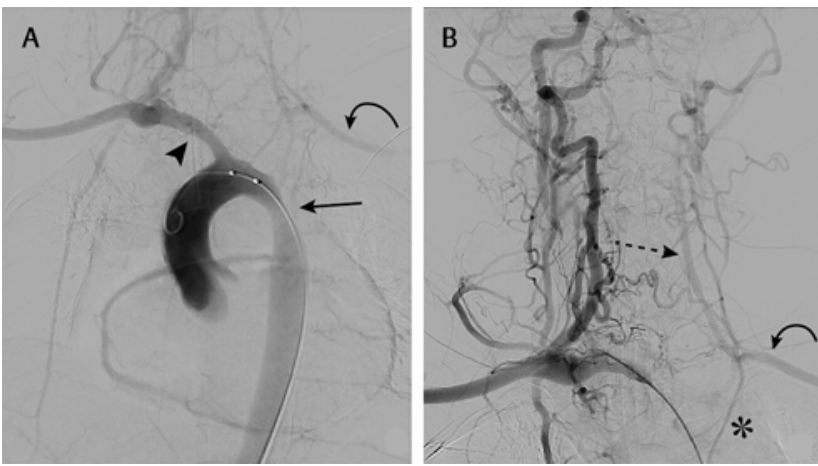

Fig. 15 Takayasu's arteritis. An 8-year-old female with diminished peripheral left upper extremity pulses. (A) LAO arch aortogram. There is smooth stenosis of the posterior aortic arch (arrow) with occlusion of the left common carotid and proximal left subclavian arteries. The brachiocephalic artery (arrowhead) is relatively preserved and serves as the only intracranial inflow. The distal subclavian artery is faintly noted (curved arrow). (B) Delayed image from brachiocephalic arteriogram. The distal left subclavian artery (curved arrow) and internal thoracic artery (asterisk) are supplied by the retrograde flow through the left vertebral artery (dashed arrow).

\section{Arterial Thoracic Outlet Syndrome}

The spectrum of symptomatic thoracic outlet syndrome includes neurogenic, venous, and arterial manifestations, of which arterial is least common. Mechanical compression by adjacent musculoskeletal structures such as a cervical rib, the clavicle, or the scalene musculature results in repetitive microtrauma to the subclavian or axillary artery, typically becoming evident with acute or chronic limb ischemia. A bruit over the subclavian artery may be found on physical examination, along with diminished ipsilateral pulses. Angiographic findings include extrinsic compression, aneurysm, dissection, in situ thrombosis, focal stenosis, poststenotic dilation, and distal embolism. Provocation with positional maneuvers during angiography, typically arm hyperabduction and external rotation with or without contralateral head rotation, confirms the diagnosis ( - Fig. 4).

\section{Thromboangiitis Obliterans (Buerger's Disease)}

Thromboangiitis obliterans (Buerger's disease) is a small- to medium-vessel non-necrotizing vasculitis, typically involving the peripheral arteries and veins of the distal upper and lower extremities. This disease most commonly affects young men with a history of smoking. Clinical presentation ranges from claudication to ischemic ulceration. Angiographic findings include extensive distal arterial occlusions classically with "corkscrew" collaterals representing the dilated vasa vasorum (-Fig. 16). Proximal large inflow vessels are preserved.

\section{Hypothenar Hammer Syndrome}

Repetitive blunt or vibratory trauma to the palm (e.g., jackhammer workers, karate enthusiasts) can cause arterial injury to the ulnar artery as it crosses the hook of hamate, with

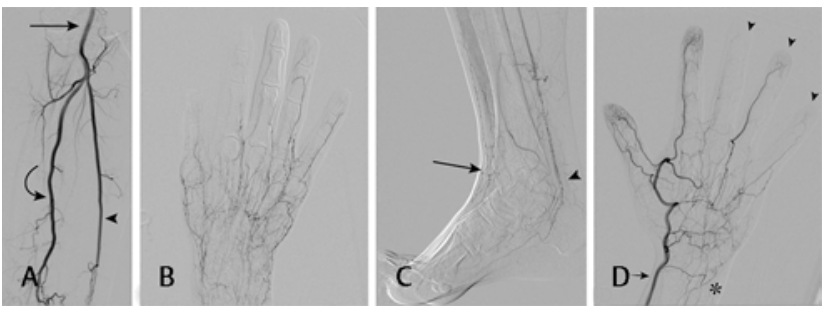

Fig. 16 Thromboangiitis obliterans (Buerger's disease). (A) Spared forearm inflow arteries. The distal brachial artery (arrow) and proximal to midradial (arrowhead) and ulnar (curved arrow) arteries are normal. The distal radial and ulnar arteries, however, demonstrate the classic "corkscrew" collaterals. (B) There is diffuse occlusion of the named arteries of the hand with extensive collateralization and poor perfusion of the digits. (C) Lower extremities are often involved as well. Multiple "corkscrew" collaterals are seen in the anterior tibial artery (arrow) and posterior tibial artery (arrowhead) territories. (D) Hand angiogram of a different patient demonstrates occlusion of the ulnar artery (asterisk), numerous tortuous collaterals within the palm, and proper digital artery occlusions in multiple fingers (arrowheads). The radial artery (arrow) is relatively preserved.

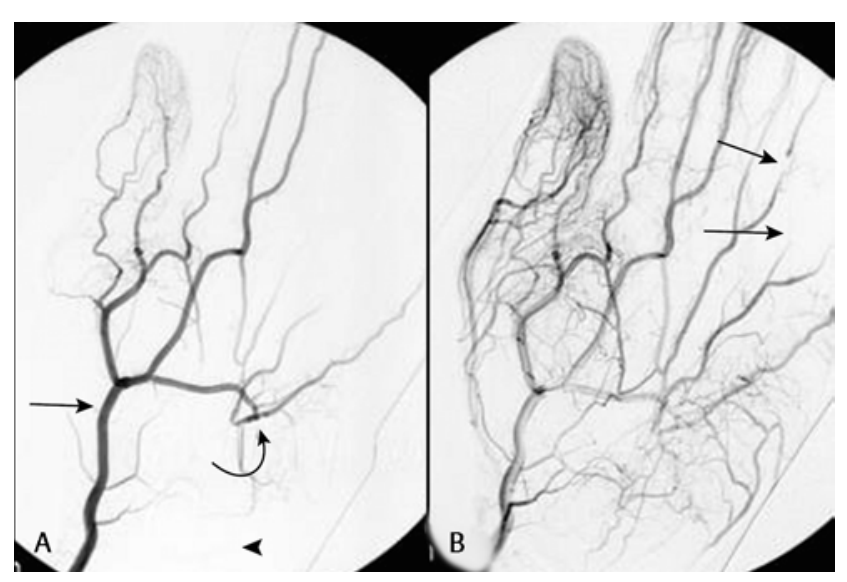

Fig. 17 Hypothenar hammer syndrome. (A) The ulnar artery is occluded (arrowhead), whereas the radial artery (arrow) is intact. Multiple microaneurysms (curved arrow) are seen over the hamate. (B) Distal occlusions of the proper digital arteries (arrows) are noted in the fourth digit.

resultant digital ischemia ("hypothenar hammer syndrome"). Angiographic findings include ulnar artery occlusion, aneurysm formation, and distal embolism ( - Fig. 17).

\section{Raynaud's Phenomenon}

Raynaud's phenomenon consists of episodic symptomatic attacks of distal small vessel vasospasm in the hands, precipitated by stimuli such as exposure to cold. Sharply demarcated skin color changes of the digits are classic. Digits initially appear white and progress to blue (a manifestation of stagnated deoxyhemoglobin), then red (reactive hyperemia), before returning to normal. ${ }^{20}$ Raynaud's phenomenon may be primary or secondary to a systemic collagen vascular process, such as lupus, Sjögren's syndrome, or scleroderma. Angiographic findings range from completely normal between episodes of vasospasm to digital artery occlusions and hypoperfusion of the finger tips during exacerbations ( - Fig. 18). 


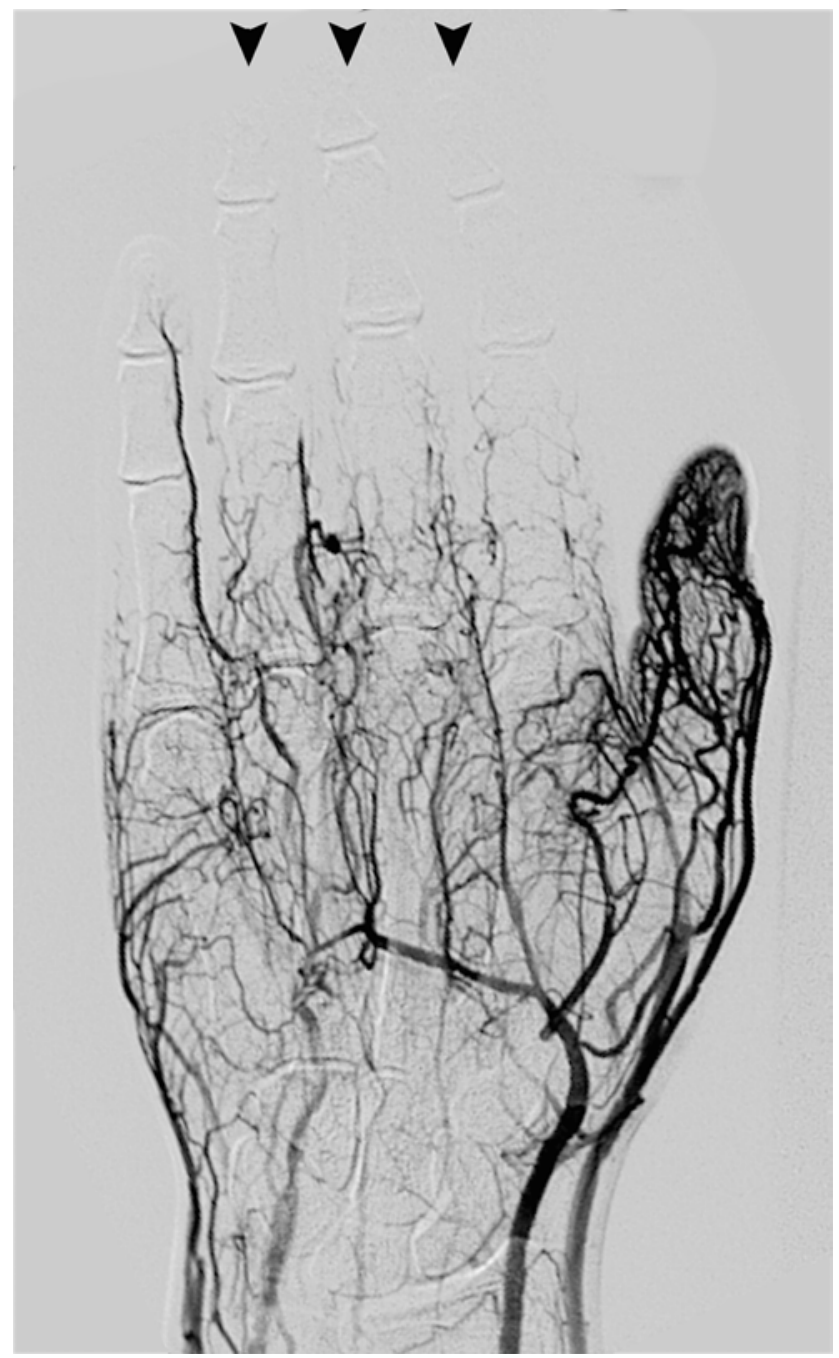

Fig. 18 Raynaud's phenomenon. Hand angiography in a patient with Raynaud's syndrome demonstrates hypoperfusion of the finger tips (arrowheads) but with relatively normal-appearing proximal vessels.

\section{Conclusion}

Although infrequently performed, catheter angiography of the upper extremity remains essential in specific clinical scenarios. Familiarity with normal and variant anatomy, basic angiographic techniques, and classic appearances of certain pathologic entities will prove useful when the need for this procedure arises.

\section{References}

1 Bozlar U, Ogur T, Norton PT, Khaja MS, All J, Hagspiel KD. CT angiography of the upper extremity arterial system: Part 1anatomy, technique, and use in trauma patients. AJR Am J Roentgenol 2013;201(4):745-752
2 Miller-Thomas MM, West OC, Cohen AM. Diagnosing traumatic arterial injury in the extremities with CT angiography: pearls and pitfalls. Radiographics 2005;25(Suppl 1):S133-S142

3 Carrafiello G, Laganà D, Mangini M, et al. Percutaneous treatment of traumatic upper-extremity arterial injuries: a single-center experience. J Vasc Interv Radiol 2011;22(1):34-39

4 Rutherford RB, Baker JD, Ernst C, et al. Recommended standards for reports dealing with lower extremity ischemia: revised version. J Vasc Surg 1997;26(3):517-538

5 Cejna M, Salomonowitz E, Wohlschlager H, Zwrtek K, Böck R, Zwrtek R. rt-PA thrombolysis in acute thromboembolic upper-extremity arterial occlusion. Cardiovasc Intervent Radiol $2001 ; 24(4): 218-223$

6 Islam A, Edgerton C, Stafford JM, et al. Anatomic findings and outcomes associated with upper extremity arteriography and selective thrombolysis for acute finger ischemia. J Vasc Surg 2014;60(2):410-417

7 Morrison HL. Catheter-directed thrombolysis for acute limb ischemia. Semin Intervent Radiol 2006;23(3):258-269

8 Verstraete M, Verhaeghe R, Belch J, et al; Working Party on Thrombolysis in the Management of Limb Ischemia. Thrombolysis in the management of lower limb peripheral arterial occlusion-a consensus document. J Vasc Interv Radiol 2003;14(9 Pt 2):S337-S349

9 Baikoussis NG, Papakonstantinou NA, Apostolakis E. Radial artery as graft for coronary artery bypass surgery: advantages and disadvantages for its usage focused on structural and biological characteristics. J Cardiol 2014;63(5):321-328

10 Leon C, Asif A. Arteriovenous access and hand pain: the distal hypoperfusion ischemic syndrome. Clin J Am Soc Nephrol 2007;2(1):175-183

11 Tordoir JH, Dammers R, van der Sande FM. Upper extremity ischemia and hemodialysis vascular access. Eur J Vasc Endovasc Surg 2004;27(1):1-5

12 Prieto-González S, Espígol-Frigolé G, García-Martínez A, et al. The expanding role of imaging in systemic vasculitis. Rheum Dis Clin North Am 2016;42(4):733-751

13 Vogelzang RL. Arteriography of the hand and wrist. Hand Clin 1991;7(1):63-86

14 Natsis KI, Tsitouridis IA, Didagelos MV, Fillipidis AA, Vlasis KG, Tsikaras PD. Anatomical variations in the branches of the human aortic arch in 633 angiographies: clinical significance and literature review. Surg Radiol Anat 2009;31(5):319-323

15 Wong VW, Katz RD, Higgins JP. Interpretation of upper extremity arteriography: vascular anatomy and pathology [corrected] Hand Clin 2015;31(1):121-134

16 Vollala VR, Jetti R, Soni S. High origin of an ulnar arterydevelopment and surgical significance. Chang Gung Med J 2011;34(6, Suppl):39-42

17 Eid N, Ito Y, Shibata MA, Otsuki Y. Persistent median artery: cadaveric study and review of the literature. Clin Anat 2011;24(5):627-633

18 Gellman H, Botte MJ, Shankwiler J, Gelberman RH. Arterial patterns of the deep and superficial palmar arches. Clin Orthop Relat Res 2001; (383):41-46

19 Johnston SL, Lock RJ, Gompels MM. Takayasu arteritis: a review. J Clin Pathol 2002;55(7):481-486

20 Phillips CS, Murphy MS. Vascular problems of the upper extremity: a primer for the orthopaedic surgeon. J Am Acad Orthop Surg 2002;10(6):401-408 